\title{
Understanding the effect of resonant magnetic perturbations on tearing mode dynamics
}

\author{
Qiming $\mathrm{Hu}^{1}$, Bo Rao ${ }^{1, \text { a) }}$, Q. Yu², Yonghua Ding ${ }^{1}$, Ge Zhuang ${ }^{1}$, Wei Jin ${ }^{1}$, Xiwei Hu${ }^{1}$ \\ 1 State Key Laboratory of Advanced Electromagnetic Engineering and Technology, \\ Huazhong University of Science and Technology, 430074, Wuhan, China \\ 2 Max-Planck-Institut für Plasmaphysik, EURATOM association, 85748 Garching, \\ Germany
}

\begin{abstract}
Numerical understandings of the effect of resonant magnetic perturbations (RMPs) on 2/1 tearing mode (TM) dynamics observed on J-TEXT tokamak (Hu Q. et al $2012 \mathrm{Nucl}$. Fusion 52 083011) are presented in this paper. The non-uniform mode rotation frequency, modulated by electromagnetic force $\left(F_{e m}\right)$ and viscous force $\left(F_{v s}\right)$, results in the applied RMPs contributing both net stabilizing and braking effect on TM. Numerical evaluation based on analytical theory shows the applied RMP contributes a dominant stabilizing effect, which are responsible for the suppression of TM. The dynamics of the first discovered small locked island (SLI) are investigated. It is found that the island is locked at the stabilizing phase and the saturated island width is less than the linear layer width. The simulated Mirnov signal indicates the small locked island is likely to be the complete suppression case observed in experiments. Associated with the application of RMP, the shape of Mirnov signal deviates from sinusoidal before mode locking, which is explained by numerical modeling. The comparisons between the numerical and experimental results are in good agreement phenomenally.
\end{abstract}

\section{INTRODUCTION}

In the past decades, resonant magnetic perturbation (RMP) fields are introduced in tokamak plasmas in order to study the interaction of such perturbations with magnetohydrodynamic (MHD) stability, ${ }^{1,2}$ and confinement of the plasma. ${ }^{3}$ Since the first RMPs experiment on PULSATOR, ${ }^{4}$ many experiments related to RMPs have been carried out. $^{1,4-18}$ The RMPs related experiments covers a broad spectrum of physics issues including the effect of RMPs on tearing mode (TM) dynamics, ${ }^{1,4-10}$ error field, ${ }^{11,}{ }^{12}$ particle transport, ${ }^{13,14}$ and mitigation or suppression of edge localized modes (ELMs). ${ }^{15-18}$ One of the results is that RMPs with moderate amplitude can suppress Mirnov oscillations effectively. ${ }^{4,5}$ It is clear from later experiments that in low-q and low-density plasmas the Mirnov oscillations can be drastically suppressed as a result of mode stabilization by RMPs. ${ }^{1}$ Recently, it is found that the suppression of Mirnov oscillation by RMPs is accompanied by a reduction both in mode magnitude and mode frequency. ${ }^{8} 9$ The RMPs effect on TM dynamics associated with mode stabilization has been investigated experimentally. ${ }^{7}$ It is believed that there should exist some mechanisms which are responsible for the suppression of magnetic island by RMPs. On the other hand, when the applied RMPs is relatively strong, mode locking can always be observed, ${ }^{9}$ and the shape of Mirnov signals turn to be strongly deviated from sinusoidal. ${ }^{6}$

To explain the changed TM dynamics caused by applied RMPs, many theoretical and numerical studies related to RMPs have been carried out. ${ }^{2,19-32}$ Some authors supposed that RMPs changed $\Delta^{\prime}$ so as to stabilize tearing instability. ${ }^{19}$ However, only the active RMPs experiment could be explained in which RMPs had a constant phase with respect to the

a) Author to whom correspondence should be addressed. Electronic mail: borao@hust.edu.cn. 
corresponding rotating magnetic island. Numerical computation show that the force caused by nonlinear interaction between RMPs and saturated rotating magnetic island influenced the Mirnov oscillations, ${ }^{6}$ and suppression of Mirnov oscillations amplitude was attributed to damping effect of tokamak resistive wall. Later research indicated that the above nonlinear force could affect the angular velocity of plasma rigid rotation, while the evolution of TM was not influenced. ${ }^{33}$ An analytical theory based on the interaction between RMPs and rotating plasma was developed, the obtained evolution equation of magnetic island shows a stabilizing term due to inertia of the plasma flow pattern around the island. ${ }^{20}$ Based on the analytical theory, practical research indicated that ion polarization current, resulted from changed plasma inertia, should be responsible for the stabilization of TM. ${ }^{1}$ While, on the other hand, the stabilizing effect by RMPs is thought to be due to plasma viscosity rather than the changed inertia. ${ }^{21}$ More generally, a basic theoretical framework is developed for the investigation of TM interaction in cylindrical geometry, ${ }^{2,23}$ the applied RMPs were found to contribute a stabilizing effect on TM, which is helpful for understanding RMPs related experiments. Besides, nonlinear numerical simulations show that strong flow shear around the island, driven by the nonlinear interaction, should be responsible for the reduction of island width by RMPs. ${ }^{34}$ Actually, a strong enough flow shear will drive Kelvin-Helmholtz instabilities. ${ }^{35}$ These different findings have enhanced the understanding of plasma response to RMPs, while further investigation is required in order to better understand the mechanism responsible for mode stabilization by RMPs.

The J-TEXT tokamak ${ }^{36}$ has been equipped with RMPs coils in order to study the plasma response to RMPs. ${ }^{37,38}$ The effect of RMPs on TM dynamics has been carried out, ${ }^{9}$ it is found that the $m / n=2 / 1$ TM can be (partly) stabilized by a moderate amplitude of RMPs ( $m$ and $n$ are the poloidal and toroidal mode numbers respectively). Numerical modeling reveals that the stabilization of the 2/1 TM by RMPs is possible for a sufficiently high ratio of plasma rotation velocity to the Alfvén speed, and a larger plasma viscosity enhances the mode stabilization by RMPs. ${ }^{9}$ Besides, when taking into account the effect of bootstrap current, the stabilization of TM by RMPs will be reduced. ${ }^{39}$ However, the mechanism responsible for the mode stabilization by RMPs is still not very clear.

In this paper, numerical understanding of the RMPs effect on $2 / 1 \mathrm{TM}$ dynamics is presented. It is found that the non-uniform mode frequency, modulated by electromagnetic force $\left(F_{e m}\right)$ and viscous force $\left(F_{v s}\right)$, results in both net stabilizing and braking effect on TM by applied RMPs, which are responsible for the stabilization of TM and reduction of mode frequency. The dynamics of the first discovered small locked island (SLI) in Ref. 9 are investigated. The SLI is found to be locked at the stabilizing phase and the saturated island width is less than the linear layer width. The simulated Mirnov signal indicates the SLI is likely to be the complete suppression case observed in experiments. Associated with the application of RMP, the shape of Mirnov signal deviates from sinusoidal before mode locking, which is explained by numerical modeling. The comparisons between the numerical and experimental results are in good agreement.

This paper is organized as follows. In section II, experimental setup and typical results that describe the RMPs effect on TM dynamic are shown, respectively. In section III, theoretical model and numerical results are presented to explain the basic features and understanding of the RMPs effect on TM dynamics. Finally, the paper is discussed and 
summarized in section IV.

\section{Experimental setup and results: effect of RMP on TM}

In this section, the experimental setup is introduced in Subsection IIA, the experimental results and analyses of the RMPs effect on TM dynamics are presented in Subsection II B-II E.

\section{A. Experimental setup}

Static RMPs generated by saddle coils have been applied on J-TEXT tokamak, ${ }^{36}$ a conventional circular cross-section tokamak with aspect ratio $R / a=1.05 \mathrm{~m} / 0.27 \mathrm{~m}(R$ and $a$ are the major and minor radius, respectively). In the present experiments, plasma current is $I_{p}$ $=180 \mathrm{kA}$, line averaged electron density for the central chord is $n_{e} \approx 1.5 \times 10^{19} \mathrm{~m}^{-3}$ and electron temperature $T_{e} \approx 800 \mathrm{eV} .{ }^{40}$ The safety factor at the plasma edge is $q_{a} \approx 3.5$, the volume averaged $\beta_{p}=\left\langle P_{e}\right\rangle /\left(B_{\theta}{ }^{2} / 2 \mu_{0}\right)=0.1$, at the plasma surface. The initial electron diamagnetic drift frequency $f_{*_{e}}$ for the $m / n=2 / 1$ tearing mode is about $1.5 \mathrm{kHz}$, and the mode propagates in the electron diamagnetic drift direction (counter plasma current).

The layout of the static RMP coil system on J-TEXT and spectrum of the generated radial magnetic field are shown in Ref. 9. Because of the special structure and flexible location of the coils, generated magnetic field can be in different spatial phase, making the generation of intrinsic error fields possible. For the RMPs applied in the present experiments, a resonant and dominant $m / n=2 / 1$ component of radial magnetic field $b_{r}$ of strength $63 \mu \mathrm{T}$ are generated at plasma edge, $r=a$, per kilo-ampere of coil current. ${ }^{9}$

In J-TEXT tokamak, TM activity is measured by 24 magnetic coils evenly spaced in a poloidal array about the boundary of the circular plasma cross-section and 8 additional magnetic coils are toroidally distributed. The oscillating magnetic signal generated by rotating magnetic islands can be detected directly using these coils and the array is employed to determine the mode structure for the dominant instability which is low $m$ mode $(m \leq 3)$ on J-TEXT. Two saddle pick-up coils are installed at opposing toroidal locations outside the vacuum vessel to detect the radial magnetic field generated by stationary islands, the signal of locked mode detector is named as $\mathrm{Br}_{\mathrm{n}=1}$.

On J-TEXT many experimental shots have been devoted to study the effect of RMPs on the $2 / 1$ TM instability. ${ }^{9}$ It is found that mode locking is likely to occur for lower rotation frequency $\left(f_{0}<\sim 5 \mathrm{kHz}\right)$. While, for a higher rotation frequency, three responses are observed by increasing the RMP amplitude: (i) partial suppression, (ii) complete suppression of $2 / 1 \mathrm{TM}$ and (iii) complete suppression followed by locked mode. These different plasma responses to RMPs sensitively depend on the TM frequency and RMP amplitude. The detailed time evolution and analysis of these different phenomena are presented as below.

\section{B. Partial suppression of TM}

When the mode frequency of $2 / 1 \mathrm{TM}$ is higher $\left(f_{0}>\sim 5 \mathrm{kHz}\right)$, stabilization of $2 / 1 \mathrm{TM}$ can be frequently observed with application of the RMP. For the plasma parameters presented in this paper, it is found that when RMPs of moderate amplitude (i.e. $3.5 \mathrm{kA}<I_{\text {coil }}<5.5 \mathrm{kA}$ or 2.2 Gauss $<b_{r}(m / n=2 / 1)<3.5$ Gauss $)$ is applied, partial suppression of the $2 / 1 \mathrm{TM}$ is observed, as shown in Fig. 1. The Mirnov signal (Fig. 1(b)) saturates at $0.1 \mathrm{~s}$, and the mode frequency 
stays approximately constant with $f_{0}=7.5 \mathrm{kHz}$ prior to application of the RMP. When RMPs is turned on and increases to $4.95 \mathrm{kA}$ at current flattop (Fig. 1(a)), the amplitude of $(2,1)$ component of $B_{\theta}$ decreases $46 \%$ (Fig. 1(c)), implying a decrease of the $2 / 1$ island width, while the mode frequency decreases from 7.5 to $5.5 \mathrm{kHz}$. When $I_{c}$ is reduced, both the mode amplitude (Fig. 1(c)) and mode frequency (Fig. 1(d)) recover to their initial state.

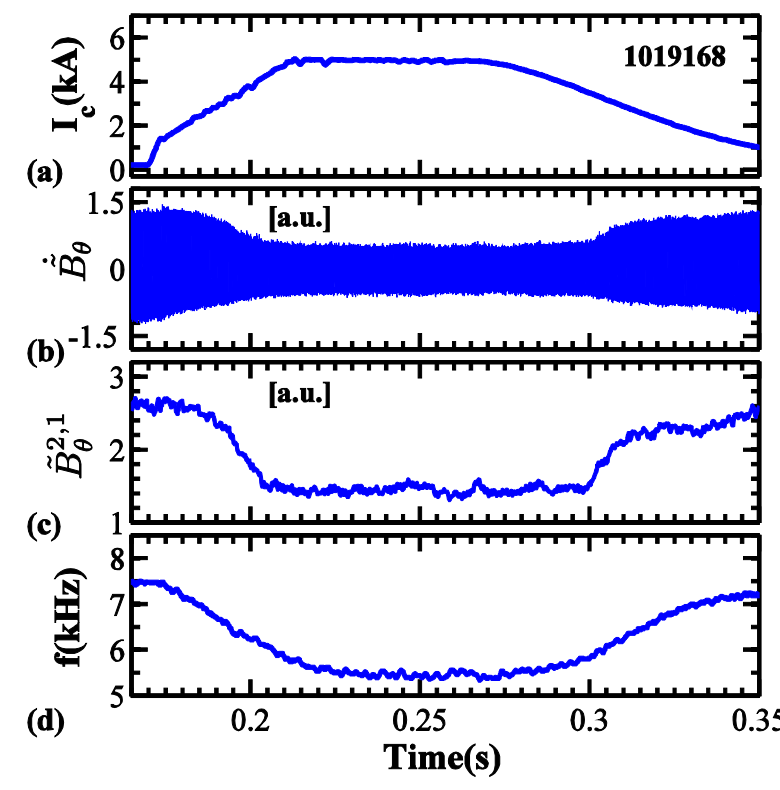

FIG. 1. Partial suppression of TM for higher mode frequency. Time evolution of (a) RMPs coils current $\mathrm{I}_{\mathrm{c}}$, (b) Mirnov signal $\mathrm{dB}_{\theta} / \mathrm{dt}$, (c) the amplitude of $(2,1)$ component of $B_{\theta}$ and $(d)$ mode frequency f analyzed by Mirnov signal.

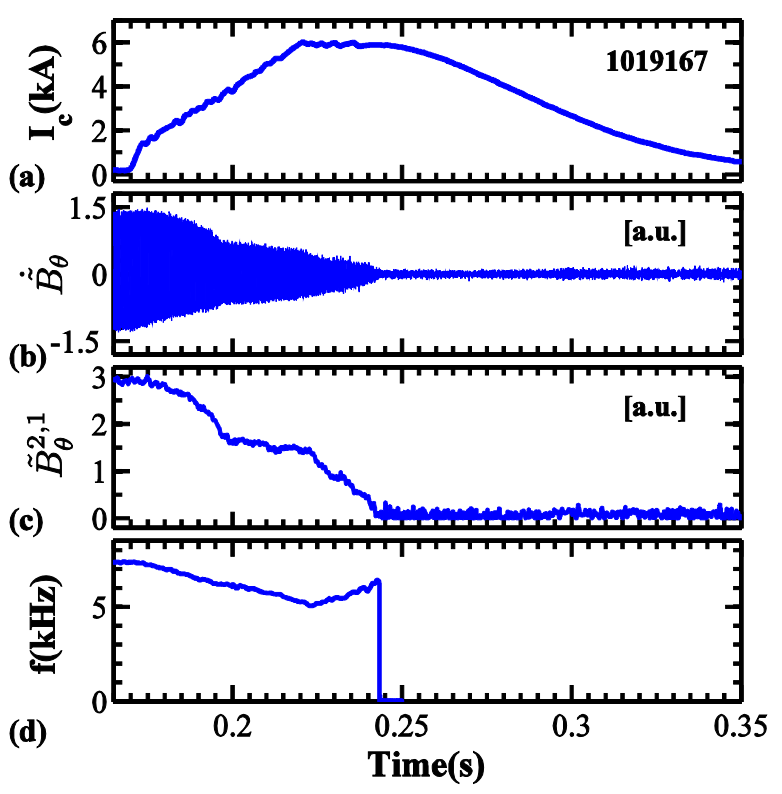

FIG. 2. Complete suppression of TM. Time evolution of (a) RMPs coils current $I_{c}$, (b) Mirnov signal $\mathrm{dB}_{\theta} / \mathrm{dt}$, (c) the amplitude of $(2,1)$ component of $\mathrm{B}_{\theta}$ and (d) mode rotation frequency f analyzed by Mirnov signal.

The stabilization of TM by RMPs has been studied previously. In Ref. 2, the ion polarization current, caused by the interaction between RMPs and TM, was thought to be responsible for the stabilization of TM. While in Ref. 23 the improved analytic model shows that the ion polarization current is destabilizing for TM, and the applied RMPs plays a stabilizing effect. It is shown in Ref. 21 that the stabilizing effect is due to the plasma viscosity rather than the changed plasma inertia. Based on the numerical modeling, the stabilizing mechanism will be given in Subsection III B.

\section{Complete suppression of TM}

By further increasing $I_{c}$ to $5.9 \mathrm{kA}$ at the current flattop (Fig. 2(a)), the Minov signal is completely suppressed into noise level at $t=0.242 \mathrm{~s}$ as shown in Fig. 2(b). We can see that $B_{\theta}{ }^{2,1}\left(\mathrm{~m} / \mathrm{n}=2 / 1\right.$ component of perturbed poloidal magnetic field) decreases with increasing $I_{c}$ (Fig. 2(c)), and at the time $0.242 \mathrm{~s}$ it decreases to near zero, comparable to noise. When examining the time evolution of the mode frequency $f$, it is observed to decrease from 7.5 $\mathrm{kHz}$ to $5.1 \mathrm{kHz}$ until the time $t=0.223 \mathrm{~s}$ (Fig. 2(d)). While between the time $0.223 \mathrm{~s}<t<$ $0.242 \mathrm{~s}, f$ increases with time. This frequency increase is likely to be caused by a weaker electromagnetic force on the island with decreasing island width. ${ }^{9}$ As the $2 / 1$ TM is completely suppressed into noise level at $t=0.242 \mathrm{~s}$, it is impossible to identify the $2 / 1 \mathrm{TM}$, 
hence the wavelet analysis shows $f$ drops from $6.3 \mathrm{kHz}$ to 0 . The $2 / 1 \mathrm{TM}$ doesn't appear again after removing the RMPs. In Ref. 2, complete suppression of $2 / 1 \mathrm{TM}$ at higher density has been observed too, the presumed interpretation of mode stabilization is the transport induced resistive profile evolution. While for results in Fig. 2, both the mode amplitude and mode frequency decrease, being different from results in Ref. 2, in which the mode stabilization is not correlated with any reduction in the mode rotation frequency.

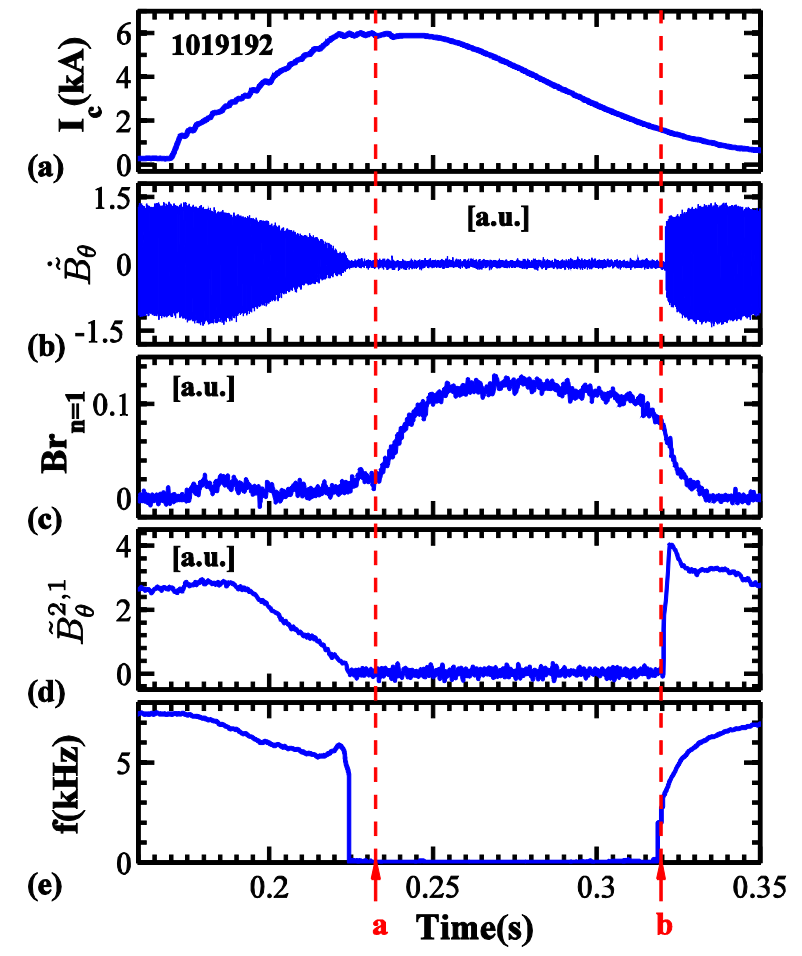

FIG. 3. Complete suppression followed by locked mode. Time evolution of (a) RMPs coils current $I_{c}$, (b) Mirnov signal $\mathrm{dB}_{\theta} / \mathrm{dt}$, (c) signal of locked mode detector $\mathrm{Br}_{\mathrm{n}=1}$, (d) the amplitude of $(2,1)$ component $\mathrm{B}_{\theta}$ and (e) mode frequency $\mathrm{f}$ analyzed by Mirnov signal.

\section{Complete suppression followed by locked mode}

When the RMP amplitude is sufficient to completely suppress the 2/1 TM as shown in Fig. 2, there is some uncertainty, sometimes locked mode is stimulated after complete suppression as shown in Fig. 3. $I_{c}$ is $5.95 \mathrm{kA}$ at the current flattop (Fig. 3(a)), and the initial mode frequency $f_{0}=7.5 \mathrm{kHz}$ (Fig. 3(e)), same as that shown in Fig. 2. The mode frequency decreases from $7.5 \mathrm{kHz}$ to $5.3 \mathrm{kHz}$, and then increases to $5.9 \mathrm{kHz}$. The time evolutions of all the signals are the same as complete suppression case as shown in Fig. 2, at $t=0.224 \mathrm{~s}$ the $2 / 1$ TM is completely suppressed (Fig. 3(b)). Then $8 \mathrm{~ms}$ later, the sudden increase in $B r_{n=1}$ (Fig. 3(c)) indicates locked mode is stimulated (marked a in Fig. 3(e)), resulting in a large locked island. When $I_{c}$ is reduced to $1.64 \mathrm{kA}$ at $t=0.319 \mathrm{~s}$ (marked b in Fig. 3(e)), the island unlocks from the applied RMPs. Different from the predicted critical rotation reduction of $50 \%$ for mode locking in Ref. 22, we have never observed such typical rotation reduction for the case shown in Fig. 3 on J-TEXT. Besides, we find that as long as the 2/1 TM can be completely suppressed by RMPs, higher RMP amplitude or a longer duration at the flattop of $I_{c}$ can result in locked mode. On EXTRAP TAR, ${ }^{7}$ the suppression or locking of $m / n=1 /-12$ TM by RMPs is observed, the high RMP amplitude case is likely to be that described in Fig. 3. 


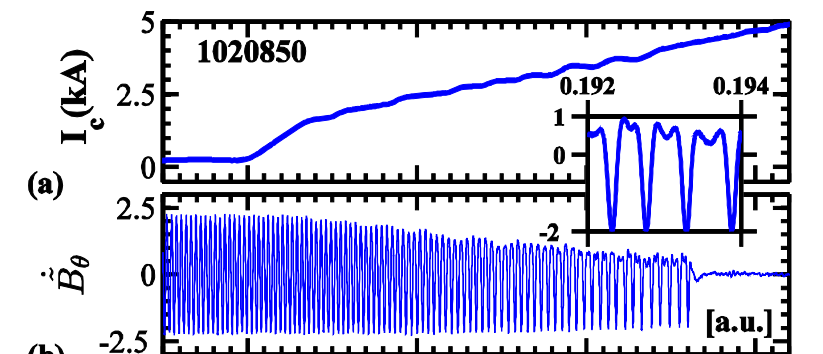

(b)

(c)

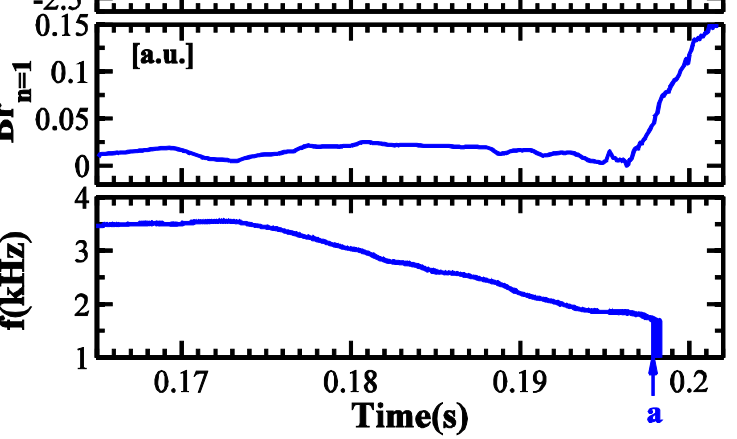

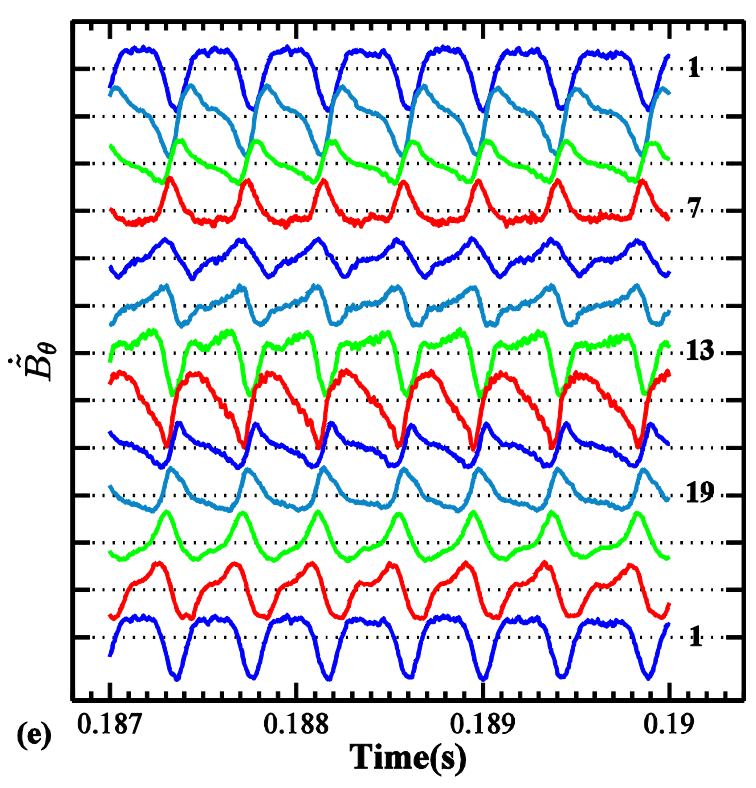

FIG. 4. Locking of 2/1 TM by RMPs for lower mode frequency. Time evolution of (a) RMPs coils current $I_{c}$, (b) Mirnov signal $d_{B} / d t$, (c) the locked mode detector signal $\mathrm{Br}_{\mathrm{n}=1}$, (d) mode rotation frequency $\mathrm{f}$ analyzed by Mirnov signal and (e) Mirnov signals from odd channels of poloidal array. The shape deviation of Mirnov signal is obviously.

\section{E. Locking of TM by RMPs}

When the mode frequency is lower, the plasma response to RMPs will be different from that shown in Subsection II B-II D. We know that locking of TM by error field or RMP requires a critical rotation reduction of $\sim 50 \%{ }^{22}$ When the mode frequency is lower, locking of 2/1 TM has been observed on J-TEXT. In Fig. 4, for example, the time evolution of $I_{c}$, $\mathrm{d} B_{\theta} / \mathrm{d} t, B r_{n=1}$ and $f$ are shown. Before the application of RMP, the plasma is undergoing a stable state and the $2 / 1 \mathrm{TM}$ saturates with the mode frequency being about $f_{0}=3.5 \mathrm{kHz}$ (Fig. 4(d)). The RMP coils are turned on at $t=0.17 \mathrm{~s}$, we can see that the mode frequency decreases as the RMP amplitude increases. Then, when $I_{c}$ increases to $4.45 \mathrm{kA}$ at $t=0.198 \mathrm{~s}$ (marked a in Fig. 4(d)), it can be seen that the signal of locked mode detector $\left(B r_{\mathrm{n}=1}\right)$ indicates the sudden growth of a large stationary mode. At the point marked a in Fig. 4(d), the mode frequency is about $f=1.76 \mathrm{kHz}$, which is consistent with the theoretical prediction in Ref. 22.

Another interesting phenomenon associated with mode locking is that the shape of Mirnov signals shows strong deviations from sinusoidal before mode locking as shown in Fig. 4(b). Detailed time evolution of $\mathrm{d} B_{\theta} / \mathrm{d} t$ during $0.192 \leq t \leq 0.194 \mathrm{~s}$ is shown at the top corner of Fig. 4(b). Actually, the deviation results from the modulation of angular velocity caused by RMPs, ${ }^{6}$ and this interpretation will be verified by numerical modeling in Subsection III E. On the other hand, this deviation of $\mathrm{d} B_{\theta} / \mathrm{d} t$ is used to evaluate error field, ${ }^{37}$ the analytic results compares favorably with experimental data. When examining the time evolution of Mirnov signals from poloidal array at different poloidal angles, the signals of odd channels are shown in Fig. 4(e). The Mirnov signal oscillation is dependent on the poloidal angle, and the crest is flattened for channels around 1 and 13, in contrast the trough is flattened for channels around 7 and 19. This phenomenon is due to the modulation of mode rotation frequency caused by RMPs as described in Subsection III E. 


\section{Theoretical model and numerical understanding}

In this section, the theoretical model is introduced in Subsection III A, the numerical understanding of the RMPs effect on TM dynamics is presented in Subsection III B-III E.

\section{A. Theoretical model}

In order to investigate the RMP effect on TM dynamics, the low $\beta$ and large aspect-ratio approximations are utilized here. The magnetic field is defined as $\boldsymbol{B}=B_{\mathrm{t}} \boldsymbol{e}_{\mathrm{t}}-(k r / m) B_{\mathrm{t}} \boldsymbol{e}_{\theta}-\nabla \psi \times \boldsymbol{e}_{\mathrm{t}}$, where $\psi$ is the helical flux function, $m / r$ and $k=n / R$ are the wave vectors in the $\boldsymbol{e}_{\theta}$ (poloidal) and the $\boldsymbol{e}_{\mathrm{t}}$ (toroidal) directions, respectively, and $m$ and $n$ are the poloidal and toroidal mode numbers.

The basic equations utilized are Ohm's law and the equation of motion. Normalizing the length to minor radius $a$, the time $t$ to the resistive time $\tau_{\mathrm{R}}=a^{2} \mu_{0} / \eta$, the helical flux $\psi$ to $a B_{\mathrm{t}}$, plasma velocity $v$ to $a / \tau_{\mathrm{R}}$, and the toroidal plasma current density $j$ to $B_{\mathrm{t}} / a$, these equations become

$$
\begin{aligned}
& \frac{\mathrm{d} \psi}{\mathrm{d} t}=E_{0}-\eta j, \\
& \frac{\mathrm{d} U}{\mathrm{~d} t}=-S^{2} \nabla_{\|} j+\mu_{\perp} \nabla_{\perp}^{2} U+S_{m},
\end{aligned}
$$

where $\mathrm{d} / \mathrm{d} t=\partial / \partial t+v_{\perp} \cdot \nabla, j=\nabla_{\perp}^{2} \psi-2 n B_{\mathrm{t}} /(m R)$ is the toroidal plasma current density. $\eta$ is the normalized plasma resistivity, and $E$ is the equilibrium electric field maintaining the equilibrium plasma current. The magnetic Reynolds number $S=\tau_{R} / \tau_{A}$, where $\tau_{A}=a / V_{A}$ is the toroidal Alfvén time. $U=-\nabla_{\perp}^{2} \phi$ is the plasma vorticity, and $\mu$ is the plasma viscosity. $S_{m}$ in equation (2) is the poloidal momentum source which leads to an equilibrium plasma rotation.

The effect of a single helicity RMP with $m / n=2 / 1$ is taken into account by the boundary condition $\psi_{2 / 1}(r=a)=\psi_{a} a B_{t} \cos (m \theta+n \varphi)$, where $\psi_{a}$ describes the normalized $m / n=2 / 1$ helical magnetic flux amplitude at $r=a, \theta$ and $\varphi$ are the poloidal and toroidal angle, respectively. The radial magnetic field perturbation at $r=a$ is given by $b_{r a}=m \psi_{a} B_{t}$. In order to study the time evolution of perturbed magnetic flux and poloidal magnetic field generated by $2 / 1 \mathrm{TM}$, 24 magnetic detectors at the radius of $r=0.85 a$ are used in the modeling. Eqs. (1) and (2) are solved simultaneously using the initial value code TM1, which has been used for modeling the nonlinear growth and saturation of $\mathrm{NTMs}^{41}{ }^{4}$ their stabilization by RF current ${ }^{27}$ and the effect of RMP on resistive TM. ${ }^{9}$

To understand the experimental results shown in Sec. II, a monotonic profile for the safety factor $q$ is taken with the $q=2 / 1$ surface located at $r_{s}=0.698 a{ }^{9}$ Based on typical experimental parameters of J-TEXT, $\tau_{R}=0.2 \mathrm{~s}, S=1 \times 10^{7}$ and $\mu_{\perp}=0.5 \mathrm{~m}^{2} \mathrm{~s}^{-1}$ are used to obtain the following numerical results as in Ref. 9. As the numerical results approximately agree with the experimental results in Ref. 9, the influence of these plasma parameters on mode stabilization caused by RMPs are further studied. Here, particularly emphasized will be understand the different RMPs effects on TM dynamic as shown in Sec. II.

\section{B. Suppression of TM}

In Fig. 5, the time evolution of normalized island width $w / a$, mode angular frequency $\omega \tau_{R}$ and simulated $\mathrm{d} B_{\theta} / \mathrm{d} t$ at the radial position of $r=0.85 a$ are shown. The amplitude of RMP 
is $\psi_{a}=4 \times 10^{-5} a B_{t}$ and it is turned on at the beginning, the initial mode angular frequency is $\omega_{0}$ $=9.5 \times 10^{3} / \tau_{\mathrm{R}}$ (corresponding to $7.5 \mathrm{kHz}$ ). It is shown that the island width is reduced from $0.05 a$ to $0.032 a$ (Fig. 5(a)), and both $\omega \tau_{R}$ (Fig. 5(b)) and $\mathrm{d} B_{\theta} / \mathrm{d} t$ (Fig. 5(c)) decrease. After the time $t=0.05 \tau_{R}, \omega \tau_{R}$ increases with time. This frequency increase is likely to be caused by weaker electromagnetic force acting on the island with decreasing island width, which will be verified later in Fig. 8. We can see that the time evolution in Fig. 5 is similar to the experimental result shown in Fig. 1. In addition, as studied in Ref. 9, a relatively higher RMP would result in a stronger TM suppression.

(a)
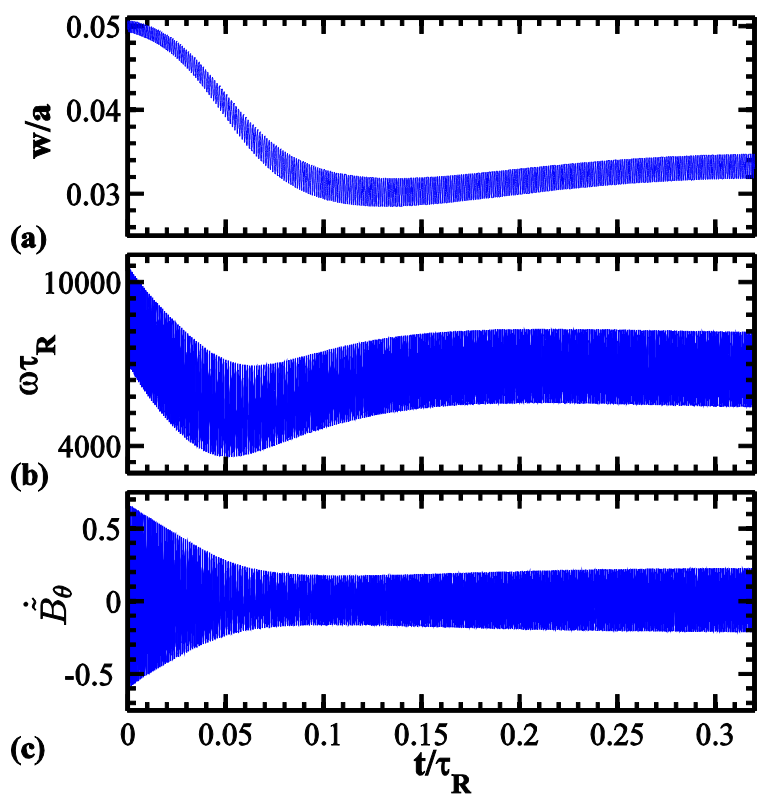

FIG. 5. Time evolution of (a) normalized island width w/a, (b) mode angular frequency $\omega \tau_{\mathrm{R}}$ and (c) simulated $\mathrm{dB}_{\theta} / \mathrm{dt}$ at the radial position of $\mathrm{r}=0.85 \mathrm{a}$. The applied RMP amplitude is $\psi_{\mathrm{a}}=4 \times 10^{-5} \mathrm{aB}_{\mathrm{t}}$, the initial mode angular frequency is $\omega_{0}=9.5 \times 10^{3} / \tau_{\mathrm{R}}$. The island width is reduced from 0.05 a to $0.032 \mathrm{a}$.

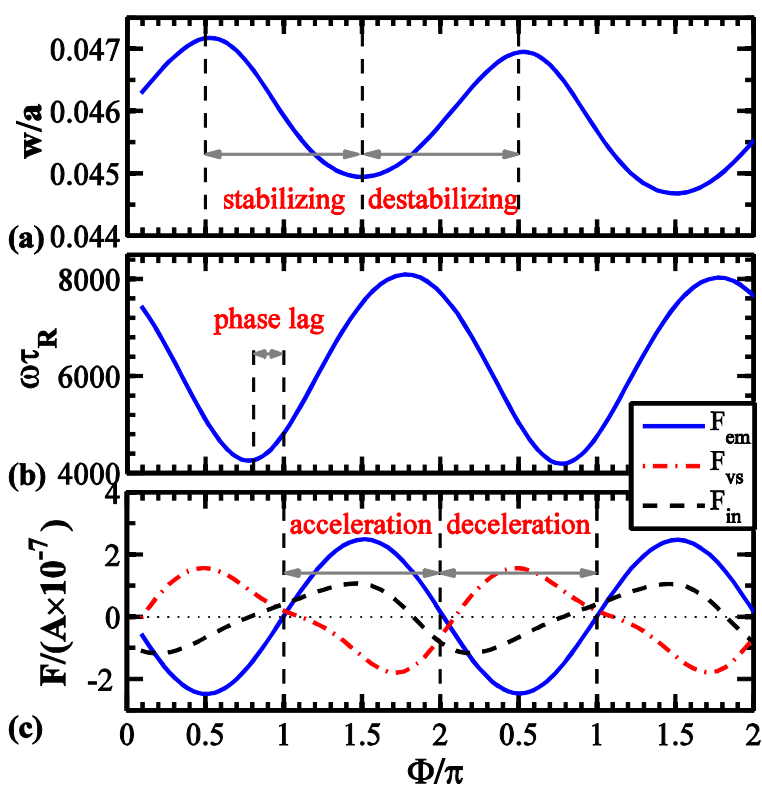

FIG. 6. Normalized (a) island width w/a, (b) mode angular frequency $\omega \tau_{\mathrm{R}}$, (c) electromagnetic force $\left(\mathrm{F}_{\mathrm{em}}\right)$, viscous force $\left(\mathrm{F}_{\mathrm{vs}}\right)$ and inertial force $\left(\mathrm{F}_{\text {in }}\right)$ at rational surface (RS) versus the relative phase $\Phi$ between island and RMP during the time $0.0296 \tau_{\mathrm{R}}$ $<\mathrm{t}<0.0317 \tau_{\mathrm{R}}$. Here, $\mathrm{A}=\mathrm{B}_{\mathrm{t}}^{2} /\left(\mathrm{a} \mu_{0}\right)$.

In order to understand the stabilizing effect contributed by the RMP, $w / a, \omega \tau_{R}$, the electromagnetic force $\left(F_{\text {em }}\right)$, the viscous force $\left(F_{v s}\right)$ and inertial force $\left(F_{\text {in }}\right)$ at the rational surface $(R S)$ versus the phase $\Phi$ during the time $0.0296 \tau_{R}<t<0.0317 \tau_{R}$ are shown in Fig. 6 . Here, $\Phi$ is the relative phase between the island and the RMP and it increases when the island rotates in the counter clock direction. $\boldsymbol{F}_{e m}=\boldsymbol{j}_{1} \times \boldsymbol{b}_{r 1}, \boldsymbol{F}_{v s}=\rho \mu \nabla^{2} \boldsymbol{v}$ and $\boldsymbol{F}_{\text {in }}=\rho \mathrm{d} \boldsymbol{v} / \mathrm{d} t$, after normalization they are $F_{e m}=A\left(j_{1} b_{r 1}{ }^{*}+j_{1}{ }^{*} b_{r 1}\right) / 2 S^{2}, F_{v s}=A \mu_{\perp} \nabla U / S^{2}$ and $F_{i n}=A / S^{2}(\mathrm{~d} v / \mathrm{d} t)$. Here $A$ $=B_{t}{ }^{2} /\left(a \mu_{0}\right), \mathrm{d} / \mathrm{d} t=\partial / \partial t+\boldsymbol{v}_{\perp} \cdot \nabla, \mu_{0}$ is the vacuum permeability and the superscript $*$ refers to the complex conjugated part. Fig. 6 shows that:

(i) Numerically, as shown in Fig. 6(a), in the phase range of $0.5 \pi<\Phi<1.5 \pi$, the RMP has a stabilizing effect on $2 / 1 \mathrm{TM}$ as the island width decreases monotonously, while in the phase range $-0.5 \pi$ (or $1.5 \pi$ ) $<\Phi<0.5 \pi$, RMP is destabilizing for $2 / 1$ $\mathrm{TM}$, which are in good agreement with the analytical theory. ${ }^{23}$

(ii) As shown in Fig. 6(c), in the phase range of $0<\Phi<\pi, F_{e m}$ is negative and contributes a deceleration to the mode rotation, while in the phase range $\pi<\Phi<2 \pi$, $F_{e m}$ is 
positive and contributes an acceleration to the mode rotation. Both $F_{v s}$ and $F_{\text {in }}$ are opposite to $F_{e m}$ at all times just with a hysteresis.

(iii) It is obvious that there is a phase lag as show in Fig. $6(\mathrm{~b}), \Delta \Phi \approx 0.2 \pi$, between the mode angular frequency $\omega \tau_{R}$ and $F_{e m}$.

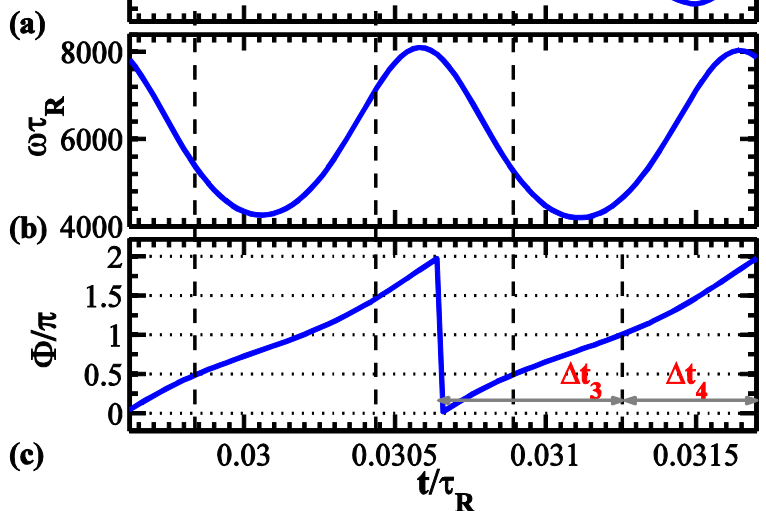

FIG. 7. Time evolution of (a) normalized island width w/a, (b) mode angular frequency $\omega \tau_{\mathrm{R}}$ and (c) phase $\Phi$ during the time $0.0296 \tau_{\mathrm{R}}<\mathrm{t}<0.0317 \tau_{\mathrm{R}}$. Here $\Delta \mathrm{t}_{1}=\Delta \mathrm{t}_{3}=6 \times 10^{-4} \tau_{\mathrm{R}}, \Delta \mathrm{t}_{2}=\Delta \mathrm{t}_{4}=4.5 \times 10^{-4} \tau_{\mathrm{R}}$.

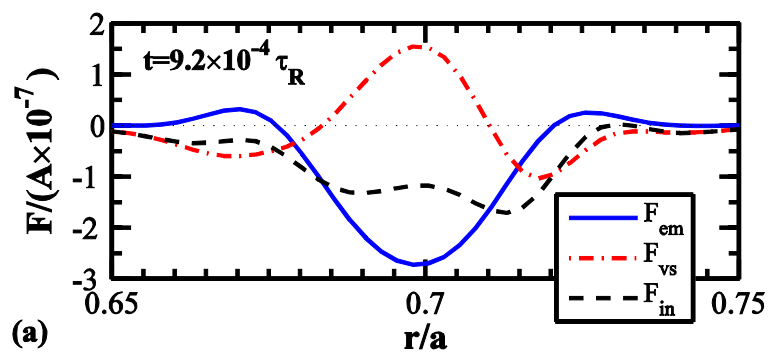

(a)

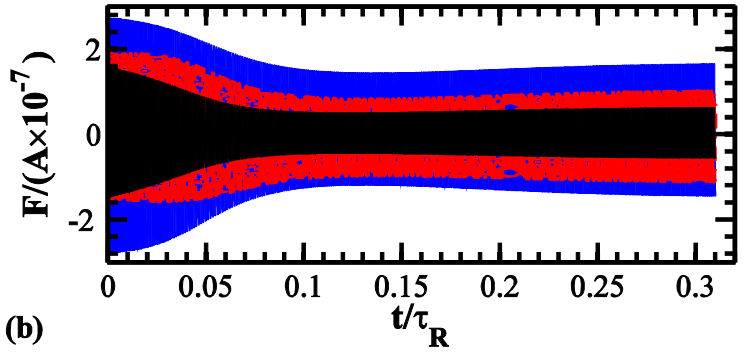

FIG. 8. (a) Radial profile of $F_{e m}, F_{v s}$ and $F_{\text {in }}$ at $t=$ $9.2 \times 10^{-4} \tau_{\mathrm{R}}$. (b) Time evolution of $F_{\mathrm{em}}, \mathrm{F}_{\mathrm{vs}}$ and $\mathrm{F}_{\text {in }}$ at RS. All the force decrease as the island width decreases.

Temporal evolution of $w / a, \omega \tau_{R}$ and phase $\Phi$ on a time scale are shown in Fig. 7. As described in Refs. 2 and 23, we can see that the mode angular frequency is modulated by $F_{e m}$ and $F_{v s}$, resulting in the island spending a slightly longer time in the stabilizing phase $(0.5 \pi<$ $\Phi<1.5 \pi$ ) than in the destabilizing phase $(-0.5 \pi$ (or $1.5 \pi)<\Phi<0.5 \pi$ ). Here, $\Delta t_{1}=6 \times 10^{-4} \tau_{R}$, $\Delta t_{2}=4.5 \times 10^{-4} \tau_{R}$, and on average the width of $2 / 1 \mathrm{TM}$ is reduced (which is evident by Fig. 7(a)). Actually, Fig. 7(a) shows a fundamental property that RMP contributes a net stabilizing effect on TM as described in Refs. 2 and 23. Another important consequence of rotation modulation by $F_{e m}$ and $F_{v s}$ is that the island spends more time in the deceleration phase $(0<\Phi$ $<\pi$, corresponding time $\left.\Delta t_{3}=6 \times 10^{-4} \tau_{R}\right)$ in which it is slowed down by the $F_{\text {em }}$ than the acceleration phase ( $\pi<\Phi<2 \pi$, corresponding time $\Delta t_{4}=4.5 \times 10^{-4} \tau_{R}$ ) in which $F_{\text {em }}$ causes it to speed up. ${ }^{23}$ This accounts for the net braking effect seen in Figs. 5 and 7, and the net braking effect explains the rotation frequency reduction shown in Figs. 1-4. The radial profile at $t=$ $9.2 \times 10^{-4} \tau_{R}$ and time evolution of $F_{e m}, F_{v s}$ and $F_{\text {in }}$ are shown in Fig. 8(a) and 8(b), respectively. The radial profiles show that all the forces are localized around $R S$. While for the time evolution, all the forces decrease with decreased island width. That is why the mode angular frequency increases after $t=0.05 \tau_{R}$. For the presented parameters, $A=0.955 \times 10^{7} \mathrm{Nm}^{-2}$, indicating $F_{\text {em }} \sim 2.5 \mathrm{Nm}^{-2}$ and $F_{v s} \sim 1.8 \mathrm{Nm}^{-2}$ at $R S$ at the first beginning, the magnitude of which are in good agreement with the experimental estimation. ${ }^{10}$

Numerical results shown in Figs. 5 to 8 indicate the applied RMP contributes a net stabilizing and braking effect to resistive TM, while it is still unable to identify what is the dominant stabilizing effect for the suppression of TM. In Ref. 23 an exhaustive island width evolu- 
tion equation has been derived as below

$$
\Delta_{t}=\Delta_{0}+\Delta_{\text {sat }}+\Delta_{R M P}+\Delta_{i o n}
$$

where $\Delta_{t}=0.8227 \tau_{R} d\left(w / r_{s}\right) / d t, \Delta_{0}$ represents the standard stability index for the $m / n=2 / 1$ tearing mode. $\Delta_{s a t}=1.6454 \lambda_{s}^{2}\left(w / r_{s}\right) \ln \left(w / r_{s}\right)$ represents the nonlinear saturation. $\Delta_{R M P}=$ $\left(w_{c} / w\right)^{2} \cos \Phi, w_{c}$ is the vacuum island width and represents the amplitude of the applied RMP. $\Delta_{i o n}$ is the destabilizing term contributed by ion polarization current, the detailed descriptions of each term in Eq. (3) refer to Ref. 23. In order to identify the dominant stabilizing effect, each term in Eq. (3) is evaluated separately based on numerical modeling as shown in Fig. 9. In Fig. 9(a), the time evolution of $\Delta_{R M P}, \Delta_{t}$ and $\Delta_{i o n}$ show that the amplitude of $\Delta_{R M P}$ is larger than that of $\Delta_{t}$, indicating the modulation of island width is mainly caused by RMP. In Fig. 9(b), more obvious results are shown by integrating each term in Eq. (3). The time evolution of $\int \Delta_{R M P} d t$ is always negative and $\left|\int \Delta_{R M P} d t\right|$ is much larger than $\left|\int \Delta_{t} d t\right|$, indicating the application of RMP contributes a dominant stabilizing effect on TM, and it should be responsible for the suppression of TM by RMP.
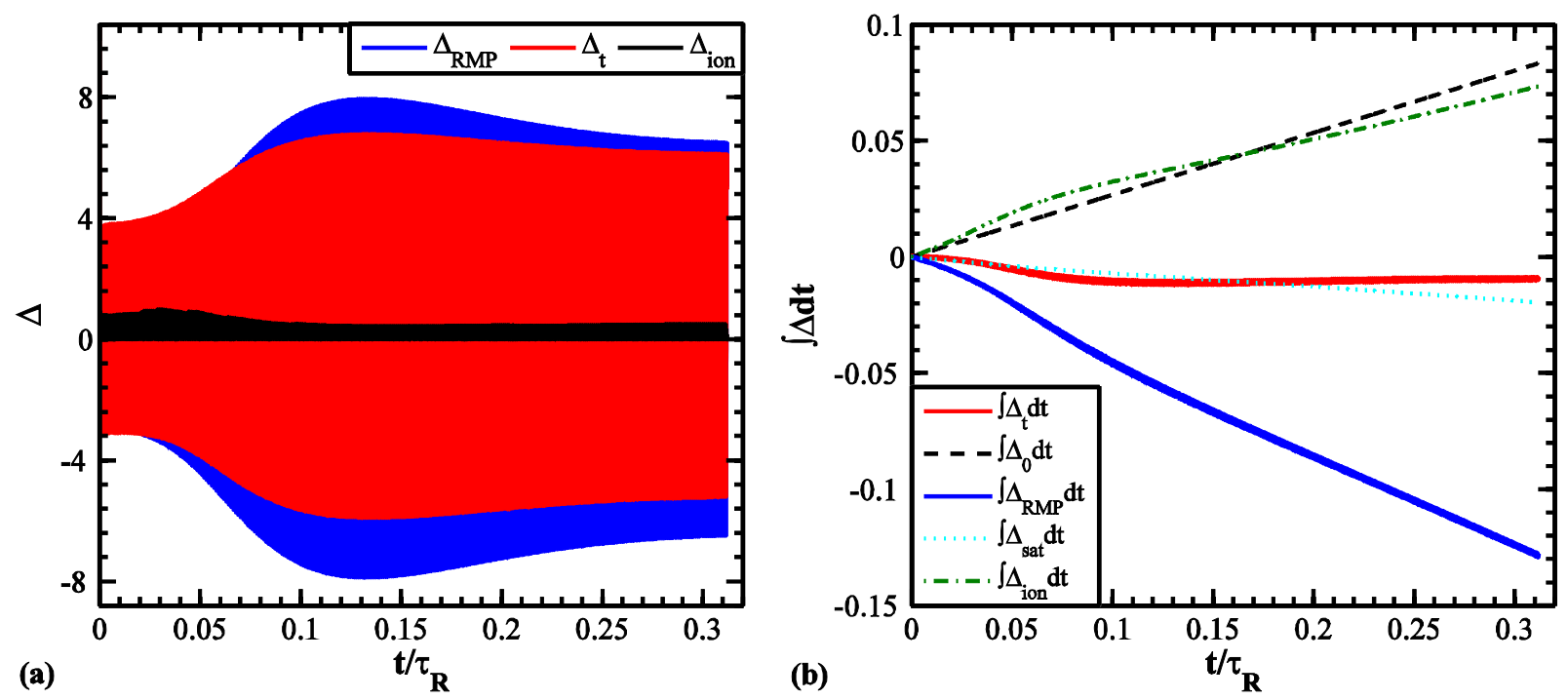

FIG. 9. (a) Time evolution of $\Delta_{\mathrm{RMP}}, \Delta_{\mathrm{t}}$ and $\Delta_{\text {ion, }}$, the amplitude of $\Delta_{\mathrm{RMP}}$ is larger than that of $\Delta_{\mathrm{t}}$. (b) Time evolution of the integral of each term in Eq. (3), the applied RMP contributes a dominant stabilizing effect on TM.

\section{Small locked island (SLI)}

When increasing the applied RMP to the amplitude of $\psi_{a}=5 \times 10^{-5} a B_{t}$, the time evolution of $w / a, 2 / 1$ mode angular frequency $\omega_{2 / 1}$ and plasma rotation angular frequency $\omega_{E}=m v_{\theta} / r_{s}$ at $R S$, simulated $\mathrm{d} B_{\theta} / \mathrm{d} t$, mode frequency $f$ analyzed by simulated $\mathrm{d} B_{\theta} / \mathrm{d} t$ and $f_{E}=\omega_{E} / 2 \pi$ (after anti-normalization) are shown in Fig. 10(a)-(d). The dashed curve shown together with $\omega_{2 / l}$ in Fig. 10(b) represents the plasma rotation angular frequency $\omega_{E}$ at $R S$. The simulated $\mathrm{d} B_{\theta} / \mathrm{d} t$ has been completely suppressed (Fig. 10(c)), while the time evolution of $\omega \tau_{R}$ (being brought to zero) shows mode locking happens. The saturated island width after mode locking is about $w=0.0119 a$, which is smaller than the viscous-resistive linear layer width ${ }^{2} \delta_{\text {layer }}=$ $2.56\left(\tau_{H}{ }^{1 / 3} \tau_{R}{ }^{1 / 6} \tau_{v}{ }^{1 / 6}\right) r_{s}=0.0245 a$ for the presented parameters. Associated with the reduction of island width, the mode angular frequency oscillates stronger and stronger, and the maximum 
turns to upwarp. Besides, when the island width decreases to less than $\delta_{\text {layer }}$ after $t=0.03 \tau_{R}$, the $2 / 1 \mathrm{TM}$ begins to decouple from plasma, which is evident by the difference between $\omega_{2 / 1}$ and $\omega_{E}$. After the time $t=0.0665 \tau_{R}$, the reversal of mode rotation appears. The wavelet analysis of simulated $\mathrm{d} B_{\theta} / \mathrm{d} t$ shows the mode frequency $f$ (dashed curve in Fig. 10(d)) decreases from $7.5 \mathrm{kHz}$ to $4.55 \mathrm{kHz}$ during the time $0<t<0.03 \tau_{R}$ and then begins to increase after $t=0.03 \tau_{R}$, and it agrees well with the plasma rotation frequency $f_{E}$ at $R S$. All the time evolution in Fig. 10 indicates the SLI is likely to be the complete suppression case shown in Fig. 2.

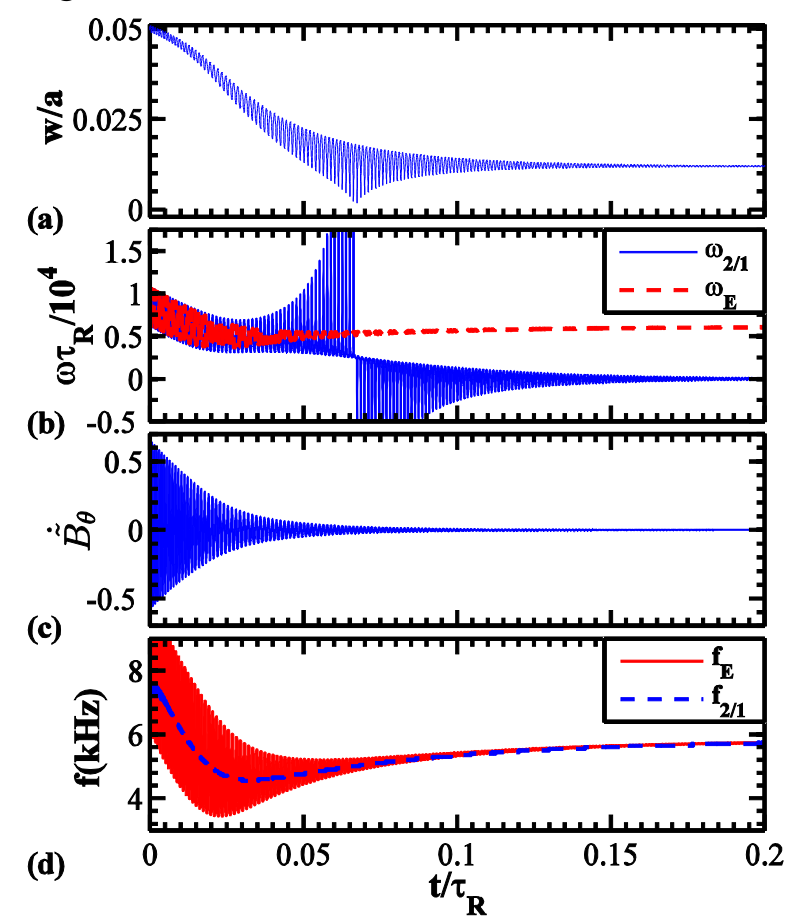

FIG. 10. Time evolution of normalized (a) island width w/a, (b) $2 / 1$ mode angular frequency $\omega_{2 / 1}$, plasma rotation angular frequency $\omega_{\mathrm{E}}=\mathrm{mv}_{\theta} / \mathrm{r}_{\mathrm{s}}$ at $\mathrm{RS}$, (c) simulated $\mathrm{dB}_{\theta} / \mathrm{dt}$ at the radial position of $\mathrm{r}$ $=0.85 \mathrm{a}$ and $(\mathrm{d})$ mode frequency $\mathrm{f}$ (dashed curve) analyzed by simulated $\mathrm{dB}_{\theta} / \mathrm{dt}$ and plasma rotation frequency (solid curve) $f_{E}=\omega_{E} / 2 \pi$ at RS. The applied RMP amplitude is $\psi_{\mathrm{a}}=5 \times 10^{-5} \mathrm{aB}_{\mathrm{t}}$, the initial mode angular frequency is $\omega_{0}=9.5 \times 10^{3} / \tau_{\mathrm{R}}$.

Corresponding to Fig. 10, the time evolution of phase $\Phi$ is shown in Fig. 11(a). We can see that before the reversal of mode rotation $\left(t<0.0665 \tau_{R}\right)$, the $2 / 1 \mathrm{TM}$ rotates in the full phase region $(0 \leq \Phi \leq 2 \pi)$. While after the reversal of mode rotation at $t=0.0665 \tau_{R}$, the $2 / 1$ TM can only rotate in the deceleration region $(0<\Phi<\pi)$. At last, the island is locked at the phase of $\Phi=0.54 \pi$. When the $2 / 1 \mathrm{TM}$ rotates in the opposite direction, all the time evolution are the same as that shown in Fig. 10 except the phase as shown in Fig. 11(b), the island is locked at the phase of $\Phi=1.46 \pi$.

In Fig. 12, the detailed time evolution of $w / a, \omega_{2 / 1}, \omega_{E}$ and phase $\Phi$ around the reversal point are shown. Because of the strong deceleration effect of RMP, the 2/1 TM rotates slowly and spends most of the time in the deceleration region, the mode angular frequency is decreased to $2.5 \times 10^{3} / \tau_{R}$, and the island width increases to nearly $0.02 a$. While in the acceleration region, the $2 / 1 \mathrm{TM}$ is quickly accelerated to high rotation frequency. Actually, the mode angular frequency is accelerated up to $2.5 \times 10^{5} / \tau_{R}$ and the island width is reduced to $1.57 \times 10^{-3} a$ before reversal. When the TM is further decelerated during the deceleration region, the rotation frequency becomes zero before rotating across the deceleration region, and then it is forced to rotate back, indicating rotation reversal occurs. After the reversal point, $F_{e m}$ is always negative and $F_{v s}$ is always positive, the $2 / 1 \mathrm{TM}$ rotates just in the deceleration region, like a simple pendulum rotates around $\Phi=0.5 \pi$ until being locked. 


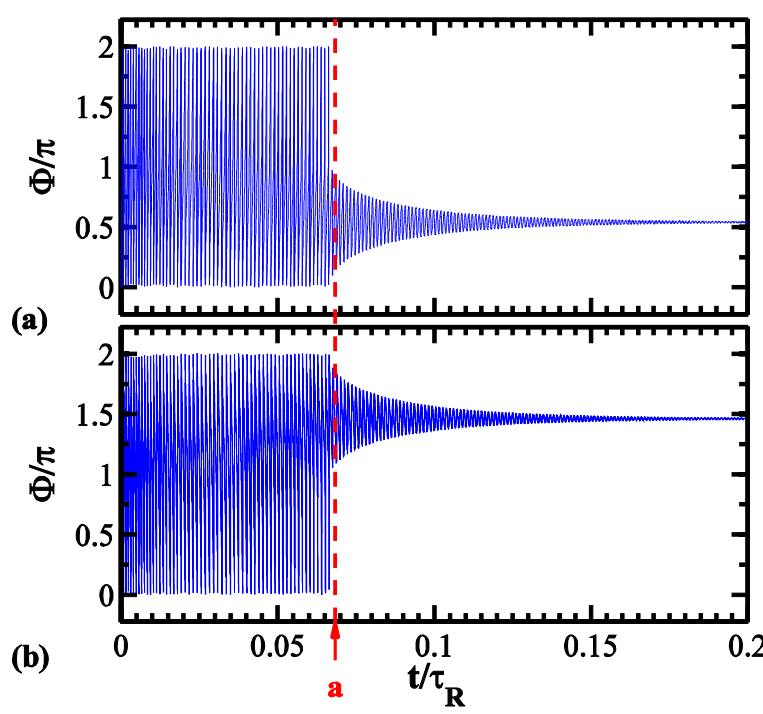

FIG. 11. Time evolution of magnetic island phase $\Phi$ for rotation direction in (a) counterclockwise and (b) clockwise.

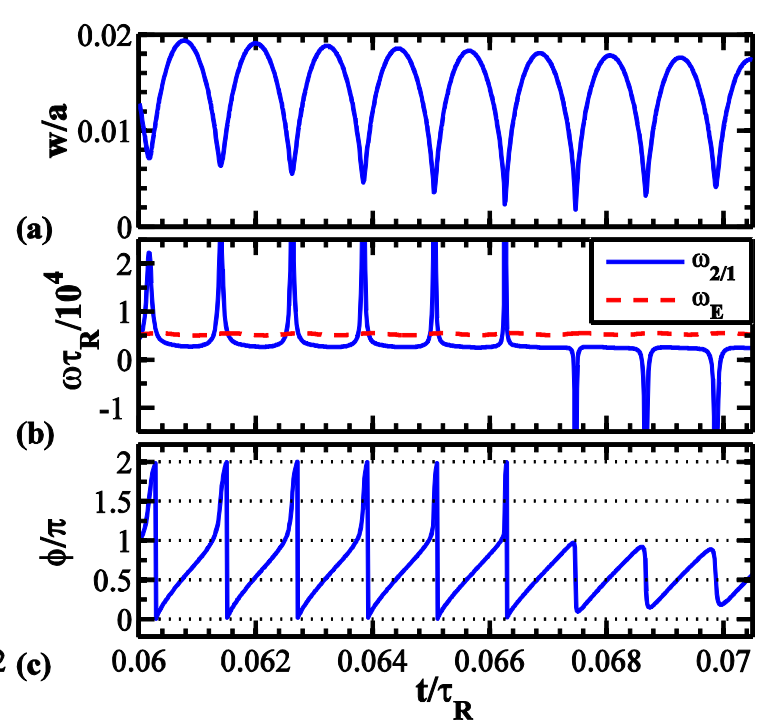

FIG. 12. Detailed time evolution of normalized (a) island width w/a, (b) mode angular frequency $\omega \tau_{\mathrm{R}}$, plasma rotation frequency $\mathrm{mv}_{\theta} / \mathrm{r}_{\mathrm{s}}$ and (c) phase $\Phi$ at $\mathrm{RS}$ for $0.06 \tau_{\mathrm{R}}<\mathrm{t}<0.07 \tau_{\mathrm{R}}$.

\section{Complete suppression followed by mode locking}

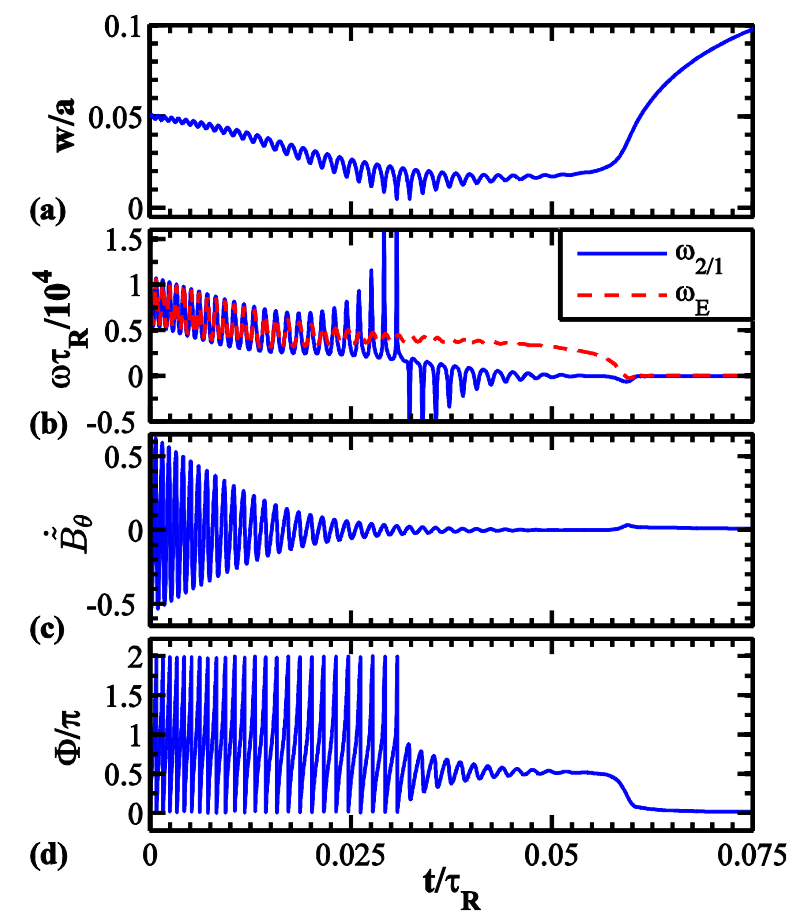

FIG. 13. Time evolution of normalized (a) island width w/a, (b) $2 / 1$ mode angular frequency $\omega_{2 / 1}$ and plasma rotation angular frequency $\omega_{\mathrm{E}}$ at $\mathrm{RS}$, (c) simulated $\mathrm{dB}_{\theta} / \mathrm{dt}$ at the radial position of $\mathrm{r}=$ $0.85 \mathrm{a}$ and (d) phase $\Phi$. The applied RMP amplitude is $\psi_{\mathrm{a}}=5.6 \times 10^{-5} \mathrm{aB}_{\mathrm{t}}, \omega_{0}=9.5 \times 10^{3} / \tau_{\mathrm{R}}$. Mode locking happens after complete suppression.

By increasing the applied RMP to the amplitude of $\psi_{a}=5.6 \times 10^{-5} a B_{t}$, the time evolution of $w / a, \omega_{2 / l}$ and $\omega_{E}$ at RS, simulated $\mathrm{d} B_{\theta} / \mathrm{d} t$ and phase $\Phi$ are shown in Fig. 13. Similar to the results shown in Figs. 10 and 11, complete suppression and reversal occurs. While after reversal point, the $2 / 1 \mathrm{TM}$ rotates in the deceleration region. After the time $t=0.05 \tau_{R}$, the phase is almost constant, then it decreases from $0.531 \pi$ to $0.5 \pi$ till $t=0.05516 \tau_{R}$ and mode locking happens at $t=0.05516 \tau_{R}$ subsequently. At last the $2 / 1 \mathrm{TM}$ is locked at the phase of $\Phi$ $=0.0067 \pi$, the island saturates with the width of $w=0.136 a$. Results shown in Fig. 13 agree 
well with the experimental results shown in Fig. 3. In Ref. 7, a full rotation of $(1,-12)$ mode is managed to rotate in the deceleration region and then locked by applied RMPs. The time evolution of TM phase and amplitude are similar to that shown in Fig. 13.

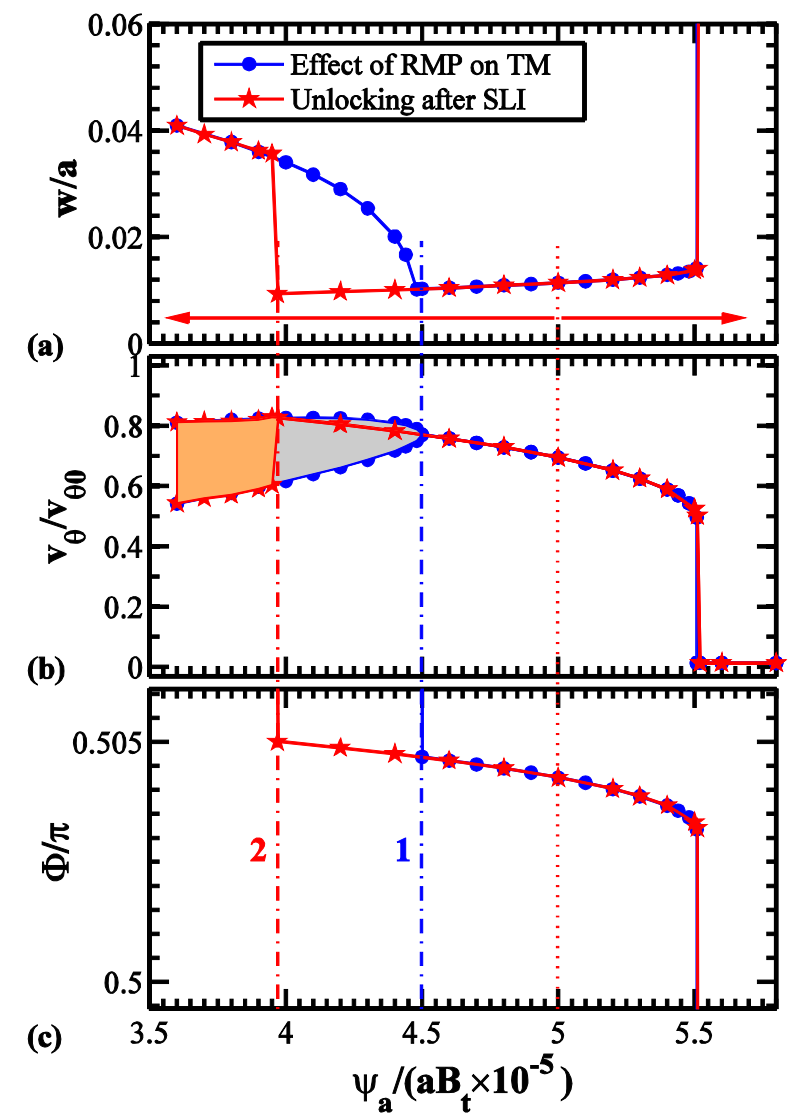

FIG. 14. The normalized (a) island width w/a, (b) poloidal rotation velocity $\mathrm{v}_{\theta} / \mathrm{v}_{\theta 0}$ and (c) the locked phase $\Phi$ versus $\psi_{\mathrm{a}}$. The solid circles correspond to general effect of RMP on TM as described in $\underline{\text { Ref. }}$ 9, the solid pentagrams correspond to results gotten by either increasing or decreasing $\psi_{\mathrm{a}}$ after SLI as the direction of arrows marked in (a). The filled area in (b) indicates the nonuniform and rotating region. Here, $\mu=0.5 \mathrm{~m}^{2} \mathrm{~s}^{-1}$ is used.

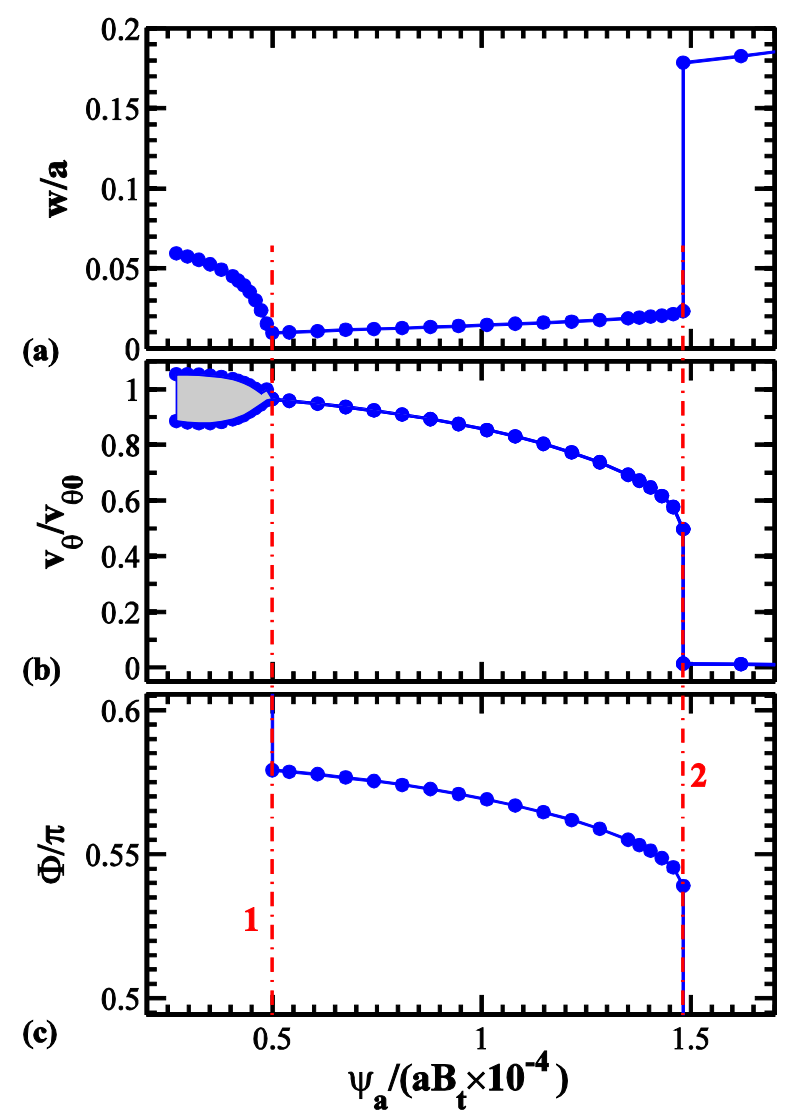

FIG. 15. The normalized (a) island width w/a, (b) poloidal rotation velocity $\mathrm{v}_{\theta} / \mathrm{v}_{\theta 0}$ and (c) the locked phase $\Phi$ versus $\psi_{\mathrm{a}}$. Here a larger plasma viscosity with $\mu=5 \mathrm{~m}^{2} \mathrm{~s}^{-1}$ is used.

In order to study the relation between SLI and the associated locked mode, the RMP effect on 2/1 TM are shown in Fig. 14 by blue solid circles. In the SLI region $\left(4.5 \times 10^{-5} a B_{t}<\right.$ $\left.\psi_{a}<5.51 \times 10^{-5} a B_{t}\right)$, the saturated island width increases from $0.01 a$ to $0.014 a$, the decoupled plasma rotation velocity decreases from $0.771 v_{\theta 0}$ to $0.496 v_{\theta 0}$, the locked phase decreases from $\Phi=0.547 \pi$ to $\Phi=0.531 \pi$, which are shown in Fig. 14(a)-(c) correspondingly. When the RMP amplitude exceeds $5.51 \times 10^{-5} a B_{t}$, mode locking happens with large island width and the locked phase is near zero. While for $\psi_{a}<4.5 \times 10^{-5} a B_{t}$ (left side of the dot-dashed line marked by 1 in Fig. 14(c)), the island is partly suppressed and rotates in the full phase region with nonuniform rotation velocity as shown by filled area in Fig. 14(b). Complete suppression followed by locked mode happens only when $v_{\theta}$ decreases from $0.765 v_{\theta 0}$ to $0.496 v_{\theta 0}$, being in 
agreement with the critical rotation reduction of $\sim 50 \%$ for usual mode locking. On the other hand, Fig. 14 shows the inexorable trend that the small locked island can not be locked in the stabilizing region when the amplitude of applied RMP is sufficiently large, then mode locking happens.

In Refs. 1 and 23, the hysteresis in the locking/unlocking cycle has been observed in both experiment and theoretical modeling. When either increasing or decreasing the amplitude of $\psi_{a}$ after SLI for the case as shown in Fig. 10, the results are shown in Fig. 14(a)-(c) by solid pentagram. Here the started point is marked by dotted line in Fig. 14, the arrows shown in Fig. 14(a) indicates the way to change the RMP amplitude. Hysteresis effect (the region between the two dot-dashed lines marked with 2 and 1) is also observed. The island unlocks until $\psi_{a}$ decreases to $3.97 \times 10^{-5} a B_{t}$, and $v_{\theta}$ increases to $0.83 v_{\theta 0}$ until mode unlocks. Actually, this hysteresis effect is less obvious than the usual mode locking or mode penetration observed in Refs. 1 and 23. Further calculations show that for the case shown in Fig. 13, mode unlocking happens until $\psi_{a}$ decreases to $1.92 \times 10^{-6} a B_{t}$, which is much smaller than $3.97 \times 10^{-5} a B_{t}$. The rotating plasma for the former case is responsible for the difference between the two hysteresis cases.

It is found in Ref. 9 that a larger plasma viscosity enhances the mode stabilization by RMP, in Fig. 15(a)-(c) the results are shown for case of plasma viscosity $\mu=5 \mathrm{~m}^{2} \mathrm{~s}^{-1}$. For $\mu=$ $5 \mathrm{~m}^{2} \mathrm{~s}^{-1}$, the SLI region is $5 \times 10^{-5} a B_{t} \leq \psi_{a} \leq 1.48 \times 10^{-4} a B_{t}$ (the region between the two dotdashed lines marked with 1 and 2), the locked phase decreases from $\Phi=0.58 \pi$ to $\Phi=$ $0.538 \pi$, and the poloidal rotation velocity at $R S$ decreases from $0.964 v_{\theta 0}$ to $0.492 v_{\theta 0}$. The results shown in Fig. 15 reveal that a larger plasma viscosity makes the SLI region much wider, and the locked phase can be larger.

\section{E. Mode locking}
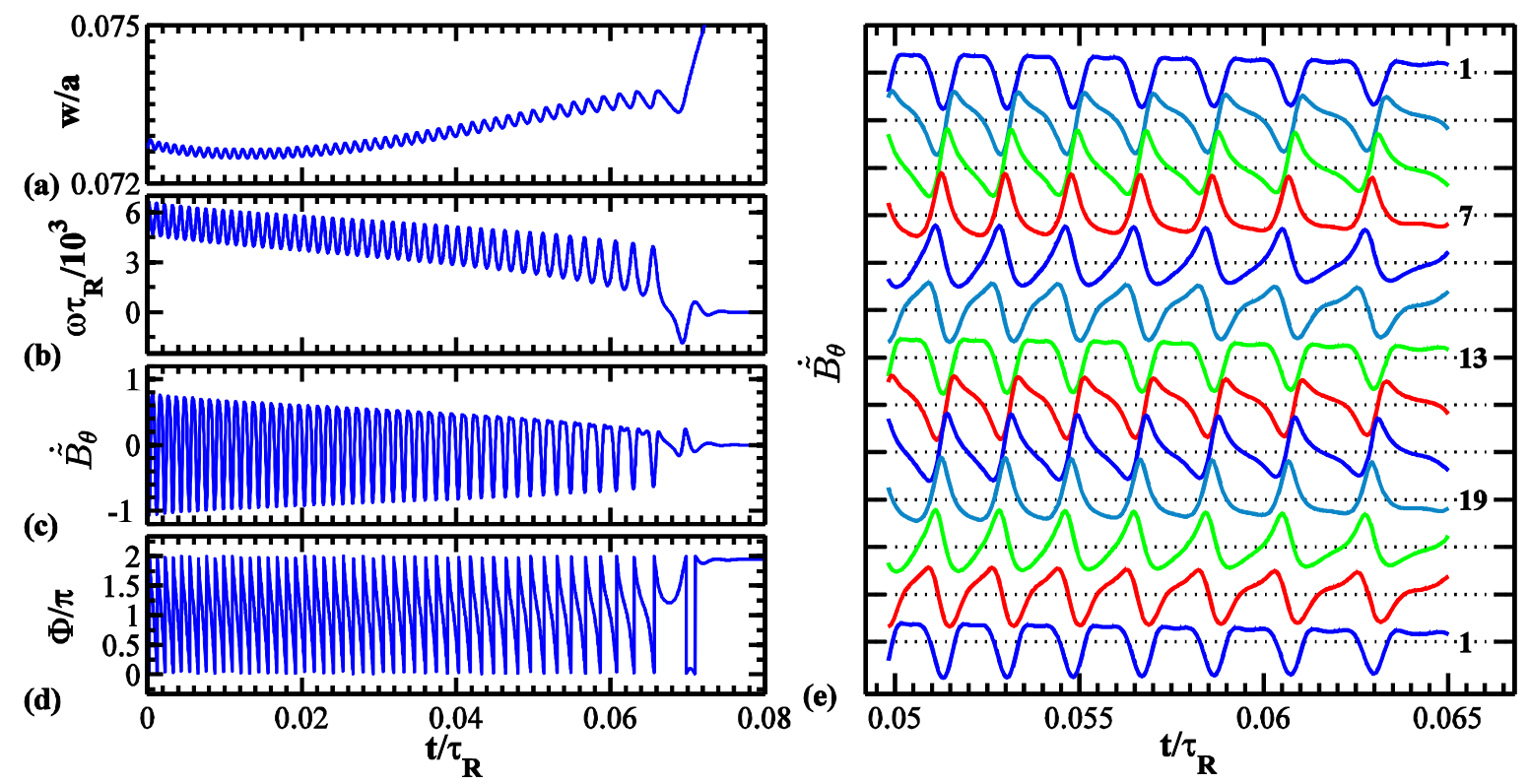

FIG. 16. Time evolution of normalized (a) island width w/a, (b) mode angular frequency $\omega \tau_{\mathrm{R}}$, (c) simulated $\mathrm{dB}_{\theta} / \mathrm{dt}$, (d) phase $\Phi$ and (e) simulated $\mathrm{dB}_{\theta} / \mathrm{dt}$ from odd channels of detector array during the time $0.05 \tau_{\mathrm{R}}<\mathrm{t}$ $<0.065 \tau_{\mathrm{R}}$. The applied RMP amplitude is $\psi_{\mathrm{a}}=6 \times 10^{-6} \mathrm{aB}_{\mathrm{t}}, \omega_{0}=5.6 \times 10^{3} / \tau_{\mathrm{R}}$, the simulated $\mathrm{dB}_{\theta} / \mathrm{dt}$ is detected at the radial position of $\mathrm{r}=0.85 \mathrm{a}$.. Mode locking happens, and the results agree well with experimental 
results shown in Fig. 4.

For a lower mode angular frequency, mode locking caused by RMP is shown in Fig. 16. The applied RMP amplitude is $\psi_{a}=6 \times 10^{-6} a B_{t}$, the initial mode angular frequency is $\omega_{0}=5.6$ $\times 10^{3} / \tau_{R}$ (corresponding to $4.5 \mathrm{kHz}$ ), the $2 / 1 \mathrm{TM}$ rotates in the clockwise direction. We can see that, during the time $0<t<0.02 \tau_{R}$, the island width decreases with time, indicating the net stabilizing effect of RMP on TM. When the mode angular frequency is obviously reduced, the island width begins to increase after $t=0.02 \tau_{R}$. At $t=0.072 \tau_{R}$ the mode angular frequency is brought to zero and mode locking happens. The shape of $\mathrm{d} B_{\theta} / \mathrm{d} t$ before mode locking shows strong deviations from sinusoidal too.

In Fig. 16(e), the time evolution of simulated $\mathrm{d} B_{\theta} / \mathrm{d} t$ from odd channels of detector array during the time $0.05 \tau_{R}<t<0.065 \tau_{R}$ are shown. Comparison between numerical and experimental results shown in Fig. 4(e) indicates they are in good agreement. The strongly modulated mode angular frequency shown in Fig. 16(b) implies the TM spends different time in both the deceleration and acceleration region as studied in Subsection III B, which is responsible for the deviation of the shape of Mirnov signals. On the other hand, the period of $\mathrm{d} B_{\theta} / \mathrm{d} t$ becomes longer, indicating the reduction of rotation or momentum loss caused by RMP as described in Ref. 6 .

\section{Discussion and summary}

Based on the reduced MHD equations, extensive numerical modeling has been carried out to understand the RMPs effect on TM dynamics and then qualitatively compared with experimental results observed on J-TEXT tokamak.

The non-uniform mode rotation frequency, modulated by $F_{e m}$ and $F_{v s}$, causes the island to spend a slightly longer time in the stabilizing phase $(-0.5 \pi<\Phi<0.5 \pi)$ than in the destabilizing phase $(0.5 \pi<\Phi<1.5 \pi)$, and on average the island experiences a net stabilizing effect by RMPs as described in Refs. 2 and 23. Another effect of rotation modulation by $F_{\text {em }}$ and $F_{v s}$ is that the island spends more time in the deceleration phase $(0<\Phi<\pi)$ than in the acceleration phase $(\pi<\Phi<2 \pi)$, resulting in a net braking effect, that is why rotation reduction is usually observed associated with mode stabilization. ${ }^{1,8,9}$ Based on the above understanding and numerical studies in Ref. 9, we conclude that the applied RMPs contributes a net and dominant stabilizing effect on TM, whether the TM can be stabilized depends on the plasma rotation and other plasma parameters. On one hand, for certain plasma parameters, when the plasma rotation exceeds the critical rotation frequency $\omega_{0 c},{ }^{9}$ stabilization of TM can be obtained by moderate amplitude of RMPs, and the reduction in rotation is significantly lower than the natural rotation frequency as shown in Figs. 1-3 ( $\Delta f \sim$ $2 \mathrm{kHz}, f_{0} \sim 7.5 \mathrm{kHz}$ ). In turn, the stabilizing effect contributed by RMP becomes stronger when the TM is suppressed to a smaller width as predicted by Eq. (3). While the electromagnetic force $F_{E M}$ exerted by RMP becomes smaller, resulting in a weaker braking effect on plasma rotation. Finally, by increasing the amplitude of RMPs, the island width becomes smaller and smaller until it reaches the linear layer width, the plasma rotation is decreased a little and then decoupling between TM and plasma flow happens. On the other hand, when the plasma rotation is lower (lower than $\omega_{0 c}$ ), associated with the application of RMPs, the net braking effect causes the rotation decrease to reach the critical rotation reduction for mode locking 
$\left(\Delta f / f_{0} \sim 50 \%\right)$, and then mode locking occurs instead of mode stabilization as shown in Figs. 4 and 16.

The SLI is first discovered numerically in Ref. 9, the dynamics of which are presented in this paper. Different from the usual mode locking as shown in Figs. 4 and 16, the SLI is found to be locked in the stabilizing phase with the saturated island width less than the linear layer width $\delta_{\text {layer }}$, and the TM decouples from the plasma flow. The predicted Mirnov signal indicates that the complete suppression of TM in experiment shown in Fig. 2 is likely to be SLI. The analysis of simulated Mirnov signal shows obvious frequency increase too.

Associated with the island being suppressed to linear layer width, the decreased island in turn reduces the transport across the island ${ }^{26}$, resulting in the plasma confinement being improved. Hence, sawteeth can emerge after complete suppression as described in Refs. 1 and 9. From this point, partial or complete suppression of TM by RMPs is beneficial to fusion reactor. While the studies, focusing on the influence of plasma parameters on mode stabilization in Refs. 9 and 39, indicates that stabilization of TM by RMPs is possible for a high ratio of plasma rotation velocity to Alfvén velocity, and the bootstrap current driven by pressure gradient will reduce the stabilizing effect.

In summary, understanding of the RMP effect on TM dynamics is presented based on numerical modeling and comparison with experimental results. The following conclusions are drawn.

(i) It is numerically found that the modulation of $F_{e m}$ and $F_{v s}$ on mode rotation, causes the island to spend a slightly longer time in the stabilizing phase $(-0.5 \pi<\Phi<0.5 \pi)$ than in the destabilizing phase $(0.5 \pi<\Phi<1.5 \pi)$, resulting in the applied RMP contributing a net stabilizing effect on TM. Numerical evaluation based on analytical theory shows the application of RMP contributes a dominant stabilizing effect on TM, which is responsible for the suppression of TM by RMPs.

(ii) The dynamics of the first discovered SLI are presented. The SLI is locked at the stabilizing phase and the saturated width is less than the linear layer width. The simulated Mirnov signal indicates the SLI is likely to be the complete suppression case observed in experiments. The hysteresis effect for SLI is different from usual mode locking. In addition, a larger plasma viscosity makes the SLI region much wider.

(iii) The simulated Mirnov signals agree well with the observed experimental results, the shape deviation from sinusoidal is explained based on dynamical analysis.

\section{Acknowledgements}

Qiming Hu thanks Dr. D. L. Brower for reviewing and correcting the manuscript. This work is supported by the Ministry of Science and Technology (Contract No. 2011GB109001, 2010GB107004, and 2008CB717805) and National Natural Science Foundation (Contract No. 10990214 and 11275080) and the Chang-Jiang scholar project of the Ministry of Education, China.

\section{References}

T. C. Hender, R. Fitzpatrick, A. W. Morris, P. G. Carolan, R. D. Durst, T. Edlington, J. Ferreira, S. J. Fielding, P. S. Haynes, J. Hugill, I. J. Jenkins, R. J. L. Haye, B. J. Parham, D. C. Robinson, T. N. Todd, M. Valovic, G. Vayakis, Nuclear Fusion 32, 2091 (1992). 
$2 \quad$ R. Fitzpatrick, Nuclear Fusion 33, 1049 (1993).

3 T. E. Evans, M. Goniche, A. Grosman, D. Guilhem, W. Hess, J. C. Vallet, Journal of Nuclear Materials 196198, 421 (1992).

4 F. Karger, H. Wobig, S. Corti, J. Gernhardt, O. Kluber, G. Lisitano, K. McCormick, D. Meisel, S. Sesnic, in Proceeding of the 5th IAEA Int. Conf. on Plasma Physics and Controlled Nuclear Fusion Energy Research, Tokyo, Japan (International Atomic Energy, Vienna, 1974), Vol. 1, p. 207.

5 J. Chen, J. Xie, Y. Huo, L. Li, Q. Zhao, G. Zhang, M. Wang, D. Guo, Q. Guo, P. Qin, G. Li, H. Fan, C. Deng, X. Tong, R. Huang, Nuclear Fusion 30, 2271 (1990).

$6 \quad$ H. Zohm, A. Kallenbach, H. Bruhns, G. Fussmann, O. Klüber, Europhysics Letters 11, 745 (1990).

7 L. Frassinetti, K. E. J. Olofsson, P. R. Brunsell, J. R. Drake, Nuclear Fusion 50, 035005 (2010).

$8 \quad$ S. Elgriw, D. Liu, T. Asai, A. Hirose, C. Xiao, Nuclear Fusion 51, 113008 (2011).

9 Q. Hu, Q. Yu, B. Rao, Y. H. Ding, X. W. Hu, G. Zhuang and J-TEXT Team, Nuclear Fusion 52, 083011 (2012).

10 L. Frassinetti, S. Menmuir, K. E. J. Olofsson, P. R. Brunsell, J. R. Drake, Nuclear Fusion 52, 103014 (2012).

11 R. J. L. Haye, A. W. Hyatt, J. T. Scoville, Nuclear Fusion 32, 2119 (1992).

12 R. J. Buttery, M. D. Benedetti, D. A. Gates, G. Yu, T. C. Hender, R. J. L. Haye, P. Leahy, J. A. Leuer, A. W. Morris, A. Santagiustina, J. T. Scoville, B. J. D. Tubbing, J. E. T. Team, C.-D. R. Team, D.-D. Team, Nuclear Fusion 39, 1827 (1999).

13 K. H. Finken, S. S. Abdullaev, M. W. Jakubowski, M. F. M. de Bock, S. Bozhenkov, C. Busch, M. von Hellermann, R. Jaspers, Y. Kikuchi, A. Kramer-Flecken, M. Lehnen, D. Schega, O. Schmitz, K. H. Spatschek, B. Unterberg, A. Wingen, R. C. Wolf, O. Zimmermann, T. Team, Physical Review Letters 98, 065001 (2007).

14 O. Schmitz, T. E. Evans, M. E. Fenstermacher, M. Lehnen, H. Stoschus, E. A. Unterberg, J. W. Coenen, H. Frerichs, M. W. Jakubowski, R. Laengner, C. L. Lasnier, S. Mordijck, R. A. Moyer, T. H. Osborne, H. Reimerdes, D. Reiter, U. Samm, B. Unterberg, D.-D. the, T. teams, Nuclear Fusion 52, 043005 (2012).

15 T. E. Evans, R. A. Moyer, P. R. Thomas, J. G. Watkins, T. H. Osborne, J. A. Boedo, E. J. Doyle, M. E. Fenstermacher, K. H. Finken, R. J. Groebner, M. Groth, J. H. Harris, R. J. La Haye, C. J. Lasnier, S. Masuzaki, N. Ohyabu, D. G. Pretty, T. L. Rhodes, H. Reimerdes, D. L. Rudakov, M. J. Schaffer, G. Wang, L. Zeng, Physical Review Letters 92, 235003 (2004).

16 Y. Liang, H. R. Koslowski, P. R. Thomas, E. Nardon, B. Alper, P. Andrew, Y. Andrew, G. Arnoux, Y. Baranov, M. Becoulet, M. Beurskens, T. Biewer, M. Bigi, K. Crombe, E. De La Luna, P. de Vries, W. Fundamenski, S. Gerasimov, C. Giroud, M. P. Gryaznevich, N. Hawkes, S. Hotchin, D. Howell, S. Jachmich, V. Kiptily, L. Moreira, V. Parail, S. D. Pinches, E. Rachlew, O. Zimmermann, Physical Review Letters 98, 265004 (2007).

17 A. Kirk, E. Nardon, R. Akers, M. Becoulet, G. De Temmerman, B. Dudson, B. Hnat, Y. Q. Liu, R. Martin, P. Tamain, D. Taylor, M. Team, Nuclear Fusion 50, 034008 (2010).

18 W. Suttrop, T. Eich, J. C. Fuchs, S. Günter, A. Janzer, A. Herrmann, A. Kallenbach, P. T. Lang, T. Lunt, M. Maraschek, R. M. McDermott, A. Mlynek, T. Pütterich, M. Rott, T. Vierle, E. Wolfrum, Q. Yu, I. Zammuto, H. Zohm, A. U. Team, Physical Review Letters 106, 225004 (2011).

19 D. A. Monticello, R. B. White, M. N. Rosenbluth, in Proceeding of the 7th IAEA Int. Conf. on Plasma Physics and Controlled Nuclear Fusion Energy Research, Innsbruck, Austria (International Atomic Energy, Vienna, 1978), Vol. 1, p. 605.

20 R. Fitzpatrick, T. C. Hender, Physics of Fluids B: Plasma Physics 3, 644 (1991). 
$21 \quad$ F. L. Waelbroeck, R. Fitzpatrick, Physical Review Letters 78, 1703 (1997).

22 R. Fitzpatrick, Physics of Plasmas 5, 3325 (1998).

23 R. Fitzpatrick, E. Rossi, E. P. Yu, Physics of Plasmas 8, 4489 (2001).

24 R. Fitzpatrick, Plasma Physics and Controlled Fusion 54, 094002 (2012).

25 F. L. Waelbroeck, I. Joseph, E. Nardon, M. Bécoulet, R. Fitzpatrick, Nuclear Fusion 52, 074004 (2012).

26 Q. Yu, Nuclear Fusion 47, 1244 (2007).

27 Q. Yu, S. Gunter, Nuclear Fusion 48, 065004 (2008).

28 Q. Yu, S. Gunter, Y. Kikuchi, K. H. Finken, Nuclear Fusion 48, 024007 (2008).

29 Q. Yu, S. Gunter, K. H. Finken, Physics of Plasmas 16, 042301 (2009).

30 Q. Yu, S. Gunter, Nuclear Fusion 51, 073030 (2011).

31 Y. Q. Liu, A. Kirk, Y. Gribov, M. P. Gryaznevich, T. C. Hender, E. Nardon, Nuclear Fusion 51, 083002 (2011).

32 Y. Q. Liu, A. Kirk, Y. Sun, P. Cahyna, I. T. Chapman, P. Denner, G. Fishpool, A. M. Garofalo, J. R. Harrison, E. Nardon, M. t. the, Plasma Physics and Controlled Fusion 54, 124013 (2012).

33 E. Lazzaro, M. F. F. Nave, Physics of Fluids 31, 1623 (1988).

34 W. Mao, M. Wang, Physics of Plasmas 1, 946 (1994).

35 I. T. Chapman, S. Brown, R. Kemp, N. R. Walkden, Nuclear Fusion 52, 042005 (2012).

36 G. Zhuang, Y. Pan, X. W. Hu, Z. J. Wang, Y. H. Ding, M. Zhang, L. Gao, X. Q. Zhang, Z. J. Yang, K. X. Yu, K. W. Gentle, H. Huang, J. T. T. the, Nuclear Fusion 51, 094020 (2011).

37 W. A. Craven, A. J. Wootton, Nuclear Fusion 38, 585 (1998).

38 B. Rao, G. Zhuang, M. Zhang, Y. H. Ding, K. X. Yu, Q. M. Hu, C. D. Hao, Y. S. Cen, B. Yi, Y. He, J. Y. Nan, IEEE Transactions on Applied Superconductivity 22, 4201804 (2012).

39 Q. Hu, Q. Yu, B. Rao, Y. H. Ding, X. W. Hu, G. Zhuang and the J-TEXT Team, "Stabilization of resistive tearing modes by applied resonant magnetic perturbations," 39th EPS Conference \& 16th Int. Congress on Plasma Physics, Stockholm, Sweden, P1. 053 (2012).

40 W. Jin, G. Zhuang, Y. H. Ding, Y. Zhang, J. Zhang, Nuclear Instruments and Methods in Physics Research Section A 674, 15 (2012).

41 Q. Yu, S. Gunter, K. Lackner, Physics of Plasmas 11, 140 (2004). 